\title{
ISOPERIMETRIC INEQUALITIES FOR CONVEX CONES
}

\author{
PIERRE-LOUIS LIONS AND FILOMENA PACELLA
}

(Communicated by Barbara L. Keyfitz)

\begin{abstract}
We present here an isoperimetric inequality for sets contained in a convex cone. Some applications to symmetrization problems and Sobolev inequalities are also indicated.
\end{abstract}

1. The aim of this note is to point out an isoperimetric inequality for convex cones which plays a fundamental role in the symmetrization of mixed boundary value problems, Sobolev inequalities, and in the understanding of the geometric properties of positive solutions of some semilinear partial differential equations.

Let $\Sigma_{\alpha}$ be an open cone in $\mathbf{R}^{n}, n \geq 2$, with vertex at the origin and solid angle $\alpha \in] 0, \omega_{n-1}\left[\right.$, where $\omega_{n-1}$ is the $(n-1)$-dimensional Hausdorff measure of the unitary sphere $S^{n-1}$. To be more precise, if $A_{\alpha}$ is a subset of $S^{n-1}$ with $H_{n-1}\left(A_{\alpha}\right)=\alpha$, then

$$
\Sigma_{\alpha}=\left\{\lambda x, x \in A_{\alpha} \text { and } \lambda \in\right] 0,+\infty[\} .
$$

From now on we will assume that $\partial \Sigma_{\alpha}$ is lipschitz continuous. We denote by $\Sigma(\alpha, R)$ the open sector in $\mathbf{R}^{n}$ with solid angle $\alpha$ and radius $R>0$, that is, $\Sigma(\alpha, R)=\Sigma_{\alpha} \cap B_{R}$ where $B_{R}$ is the ball in $\mathbf{R}^{n}$ with center in the origin and radius $R$.

The Lebesgue measure of a measurable set $E \subseteq \mathbf{R}^{n}$ will be denoted by $|E|$, while $P_{\Omega}(E)$ will represent the De Giorgi perimeter of $E$ relative to an open set $\Omega[5,2]$. In other words

$$
P_{\Omega}(E)=\sup \left\{\left|\int_{E} \operatorname{div} \psi d x\right|, \psi \in\left[C_{0}^{\infty}(\Omega)\right]^{n},|\psi| \leq 1\right\},
$$

that is, $P_{\Omega}(E)$ is the total variation on $\Omega$ of the characteristic function of the set $E$.

We will use the symbol $\alpha_{n}$ to mean the measure of any unitary sector $\Sigma(\alpha, 1)$ with solid angle $\alpha$.

Received by the editors September 21, 1988 and, in revised form, June 5, 1989.

1980 Mathematics Subject Classification (1985 Revision). Primary 52I40, 49F10.

Key words and phrases. Isoperimetric inequalities, convex cones, Brunn-Minkowski inequality.

The first author's work was partially supported by US Army Contract DASA 45-88-C-009.

The second author's work was supported by a NATO-CNR followship. 
Our main result is the following.

Theorem 1.1. If $\Sigma_{\alpha}$ is a convex cone in $\mathbf{R}^{n}, n \geq 2$, then the following isoperimetric inequality holds:

$$
P_{\Sigma_{\alpha}}(E) \geq n \alpha_{n}^{1 / n}|E|^{(n-1) / n},
$$

for any measurable set $E \subset \Sigma_{\alpha}$ with $|E|<+\infty$. Moreover if $\partial \Sigma_{\alpha} \backslash\{P\}(P$ vertex of $\Sigma_{\alpha}$ ) is smooth, equality in (1.1) holds if and only if $E$ is a convex sector $\Sigma(\alpha, R)$ homothetic to $\Sigma_{\alpha}$.

Remark 1.1. If $n=2$ the relative isoperimetric inequality (1.1) is already known (see [3, Chapter 1, Theorem 11]). If $\alpha=2 \pi / m, m$ a positive integer, it is an easy consequence of the classical isoperimetric inequality in the plane. In the case $n=2$ the only convex cones $\Sigma_{\alpha}$ are the sets $\Sigma_{\alpha}=\{(x, y)=$ $\left.(\rho, v) \in \mathbf{R}^{2}, \rho>0,0<v<\alpha\right\}$ with $\left.\left.\alpha \in\right] 0, \pi\right]$, where $(\rho, v)$ are the polar coordinates in the plane.

A higher dimensional result equivalent to that of Theorem 1.1 has been proved in [15, Proposition 1.1] only for a particular type of convex sectors $\Sigma_{\alpha}$.

To obtain the general result in $\mathbf{R}^{n}$, we adapt to our case a proof of the classical isoperimetric inequality which is based on the Brunn-Minkowski inequality (see $[7,14])$ that we are going to recall later. Such a method is classical and extensively used in [14]. Let us observe that there are several other (perhaps better known) proofs of the classical isoperimetric inequality based on symmetrization techniques $([6,11])$ which, however, do not seem to be useful in our problem.

Before proving Theorem 1.1 we need to state some preliminary lemmata. Let us denote by $A+B$ the algebraic sum of two sets in $\mathbf{R}^{n}$, i.e., $A+B=$ $\{a+b, a \in A, b \in B\}$.

Lemma 1.1 (Brunn-Minkowski's inequality). If $A$ and $B$ are measurable subsets of $\mathbf{R}^{n}$ with $|A|>0$ and $|B|>0$, then

$$
|A+B|^{1 / n} \geq|A|^{1 / n}+|B|^{1 / n} \text {. }
$$

The proof of (1.2) can be found in [7] or [14].

Let $A$ and $C$ be two measurable subsets of $\mathbf{R}^{n}$. The "exterior Minkowski relative surface of $A$ "with respect to $C$ " is defined by [14, Chapter 5]

$$
F_{+}(A, C)=\liminf _{\rho \rightarrow 0+} \frac{|A+\rho C|-|A|}{\rho},
$$

where $\rho C=\{\rho y, y \in C\}, \rho>0$.

Lemma 1.2. If $A$ and $C$ are measurable sets in $\mathbf{R}^{n}$ and $0<|A|<+\infty$, $0<|C|<+\infty$ then

$$
F_{+}(A, C) \geq n|A|^{(n-1) / n}|C|^{1 / n} .
$$

Moreover equality holds in (1.4) if and only if $A$ and $C$ are homothetic convex sets. 
Proof. This lemma is proved in [14, pp. 189-195]. The inequality (1.4) is just a consequence of (1.2). In fact, from (1.2) we get

$$
|A+\rho C| \geq\left.|| A\right|^{1 / n}+\left.\rho|C|^{1 / n}\right|^{n} \geq|A|+n|C|^{1 / n} \rho|A|^{(n-1) / n},
$$

from which (1.4) follows. The remaining part of the proof is quite complex and we refer to [14] for it.

Proof of Theroem 1.1. Inequality (1.1) is obviously true for any set $E \subset \mathbf{R}^{n}$ with $|E| \rightarrow+\infty$ and $P_{\Sigma_{r}}(E)=+\infty$, so that we can assume $P_{\Sigma_{\alpha}}(E)>+\infty$. By a property of De Giorgi's perimeter any set $E$ with finite perimeter in $\Sigma_{\alpha}$ can be approximated by a sequence of sets $E_{i}$ with smooth boundary in $\Sigma_{\alpha}$ (for example, of class $C^{1}$ ) such that $\left|E_{i}\right| \rightarrow|E|$ and $H_{n-1}\left(\partial E_{i} \cap \Omega\right) \rightarrow P_{\Omega}(E)$ (see [2] or [16, Theorem 6.1.3]).

Thus it is enough to prove (1.1) for sets $E$ such that $\partial_{\alpha} E=\partial E \cap \Sigma_{\alpha}$ is smooth.

For such sets we have ([2] and [7, Chapter 3])

$$
\begin{aligned}
P_{\Sigma_{\alpha}}(E) & =H_{n-1}\left(\partial_{\alpha} E\right) \\
& \geq \liminf _{\rho \rightarrow 0+} \frac{\left|\left\{x \in \Sigma_{\alpha}: d\left(x, \partial_{\alpha} E\right)<\rho\right\}\right|}{2 \rho},
\end{aligned}
$$

where $d(x, A)$ is the distance of the point $x$ from the set $A$.

The lim inf in the right-hand side of (1.5) is a slight variant of the so-called $(n-1)$-dimensional lower Minkowski content of $\partial_{\alpha} E$ and we will denote it by $\mathscr{M}_{*}^{n-1}\left(\partial_{\alpha} E\right)$.

For $0<\rho<+\infty$ the sets $E_{\rho}^{\prime}=\left\{x \in \Sigma_{\alpha}: 0<d(x, E)<\rho\right\}$ and $E_{\rho}^{\prime \prime}=\{x \in$ $\left.\Sigma_{\alpha}: d\left(x, \Sigma_{\alpha} \backslash E\right)<\rho\right\}$ are disjoint subsets of $\left\{x \in \Sigma_{\alpha}: d\left(x, \partial_{\alpha} E\right)<\rho\right\}$, so that from (1.5) we get

$$
H_{n-1}\left(\partial_{\alpha} E\right) \geq \liminf _{\rho \rightarrow 0+} \frac{\left|E_{\rho}^{\prime}\right|}{2 \rho}+\liminf _{\rho \rightarrow 0+} \frac{\left|E_{\rho}^{\prime \prime}\right|}{2 \rho} .
$$

Moreover, since $\Sigma_{\alpha}$ is a convex cone (hence $\Sigma_{\alpha}+\Sigma_{\alpha}=\Sigma_{\alpha}$ ) we have $E_{\rho}^{+}=$ $\left\{x \in \Sigma_{\alpha}: d(x, E)<\rho\right\} \supseteq\left(E \cup \partial_{\alpha} E\right)+\Sigma(\alpha, \rho)$ and $E_{\rho}^{\prime}=E_{\rho}^{+} \backslash\left(E \cup \partial_{\alpha} E\right)$, $E_{\rho}^{\prime} \subset E_{\rho}^{+}$. Thus, by Lemma 1.2 applied to the sets $E \cup \partial_{\alpha} E$ and $\Sigma(\alpha, 1)=$ $(1 / \rho) \Sigma(\alpha, \rho)$ we obtain

$$
\begin{aligned}
\liminf _{\rho \rightarrow 0+} \frac{\left|E_{\rho}^{\prime}\right|}{\rho} & \geq \liminf _{\rho \rightarrow 0+} \frac{\left|\left(E \cup \partial_{\alpha} E\right)+\rho \Sigma(\alpha, 1)\right|-\left|E \cup \partial_{\alpha} E\right|}{\rho} \\
& =F_{+}(E, \Sigma(\alpha, 1)) \geq n|E|^{(n-1) / n} \alpha_{n}^{1 / n} .
\end{aligned}
$$

On the other hand $E_{\rho}^{\prime \prime}=E \backslash\left\{x \in \Sigma_{\alpha}: d\left(x, \Sigma_{\alpha} \backslash E\right) \geq \rho\right\}=E \backslash E_{\rho}^{-}$and $E_{\rho}^{-}+$ $\Sigma(\alpha, \rho) \subset E$, because $\Sigma_{\alpha}$ is a convex cone. Thus applying Brunn-Minkowski's inequality as in Lemma 1.2 we get

$$
|E| \geq\left|E_{\rho}^{-}\right|+n\left|E_{\rho}^{-}\right|^{(n-1) / n} \alpha_{n}^{1 / n} \rho .
$$


Therefore

$$
\liminf _{\rho \rightarrow 0+} \frac{\left|E_{\rho}^{\prime \prime}\right|}{\rho} \geq \liminf _{\rho \rightarrow 0+} n \alpha_{n}^{1 / n}\left|E_{\rho}^{-}\right|^{(n-1) / n}=n \alpha_{n}^{1 / n}|E|^{(n-1) / n}
$$

since $\left|E_{\rho}^{-}\right| \rightarrow|E|$, as $\rho \rightarrow 0$.

From (1.6), (1.7), and (1.8) we deduce (1.1). Finally it is trivial to check that all the previous inequalities become equalities when $E$ is any sector homothetic to $\Sigma_{\alpha} \cap B_{1}$ so that $(1.1)$ is proved.

Moreover, in view of Lemma 1.2 and (1.5)-(1.7), we can immediately say that the sectors $\Sigma(\alpha, R)$ are the only sets to achieve equality in (1.1) among all the measurable subsets of $\Sigma_{\alpha}$ such that $P_{\Sigma_{\alpha}}(E) \geq \mathscr{M}_{*}^{n-1}\left(\partial_{\alpha} E\right)$. Therefore, to prove the uniqueness statement it is enough to show that for any optimal set $F$ the following relation holds: $P_{\Sigma}(F) \geq \mathscr{M}_{*}^{n-1}\left(\partial_{\alpha} F\right)$.

Let us start by observing that $F$ achieves equality in (1.1) if and only if $F$ minimizes the perimeter $P_{\Sigma_{\alpha}}(E)$ among all the sets with fixed measure $|E|=$ $|F|$. Then, by some regularity results $([10,12,8,13])$ we have

(i) $\partial_{\alpha} F$ is the union of a $C^{1}$-manifold and a closed singular set whose $s$-dimensional Hausdorff measure is zero, for every $s>n-8$;

(ii) for almost every point $x$ in $\Sigma_{\alpha}$ the segment of minimal length from $x$ to $\partial_{\alpha} F$ reaches $\partial_{\alpha} F$ at a regular point;

(iii) if $\partial \Sigma_{\alpha} \backslash\{P\}$ is smooth, $\partial_{\alpha} F$ intersects $\partial \Sigma_{\alpha} \backslash P$ orthogonally.

Let us denote by $\partial_{\alpha}^{*} F$ the regular part of $\partial_{\alpha}(F)$; usually $\partial_{\alpha}^{*}(F)$ is called the "reduced boundary". By a property of the De Giorgi perimeter $P_{\Sigma_{\alpha}}(F)=$ $H_{n-1}\left(\partial_{\alpha}^{*} F\right)[2,16]$. Moreover by (ii), (iii) and the definition of $\mathscr{M}_{*}^{n-1}\left(\partial_{\alpha} F\right)$ we have

$$
\mathscr{M}_{*}^{n-1}\left(\partial_{\alpha} F\right)=\mathscr{M}_{*}^{n-1}\left(\partial_{\alpha}^{*} F\right) \leq H_{n-1}\left(\partial_{\alpha}^{*} F\right)=P_{\Sigma_{\alpha}}(F) .
$$

The inequality in (1.9) follows from (iii) and the smoothness of $\partial_{\alpha}^{*} F$, as for (1.5). By the definition of $\mathscr{M}_{*}^{n-1}\left(\partial_{\alpha} F\right)$, such inequality also holds when $P \in$ $\partial_{\alpha} F$, since $\Sigma_{\alpha}$ is a convex cone. From (1.9) the assertion follows.

Remark 1.2. The classical isoperimetric inequality follows immediately from Lemma 1.2. In fact for smooth measurable sets $E$ in $\mathbf{R}^{n}$ we have that $H_{n-1}(\partial E)=F_{+}\left(E, B_{1}\right)$, so that (1.4) yields the inequality

$$
P(E) \geq n C_{n}^{1 / n}|E|^{(n-1) / n}, \quad C_{n}=\left|B_{1}\right| .
$$

Since the convex set $C=\Sigma(\alpha, 1)$ we use in our proof is not a ball we cannot in general claim that $H_{n-1}\left(\partial_{\alpha} E\right)=F_{+}(E, \Sigma(\alpha, 1))$. Hence inequality (1.1) is not a direct consequence of (1.4).

Remark 1.3. The isoperimetric inequality (1.1) does not hold without any assumption on the cone $\Sigma_{\alpha}$. To suport this claim let us consider the particular cone $S_{\beta}=\left\{x \in \mathbf{R}^{n}: \rho>0, v_{i} \in\right] 0, \pi[$ for $i=1, \ldots, n-2$ and 
$\left.v_{n-1} \in\right] 0, \beta[\}$, where $v_{n-1} \in[0,2 \pi[$ and $\beta \in] 0,2 \pi[$. The boundary of $S_{\beta}$ is $\partial S_{\beta}=\left\{x \in \mathbf{R}^{n}: v_{n-1}=0\right.$ or $\left.v_{n-1}=\beta\right\}$, that is, $\partial S_{\beta}$ lies in the union of two hyperplanes $T_{0}$ and $T_{\beta}$.

Let $B\left(x_{0}, r\right)$ be a ball with center in a point $x_{0} \in \partial S_{\beta} \backslash\left(T_{0} \cap T_{\beta}\right)$ and radius $r>0$ so small that the set $D=B\left(x_{0}, r\right) \cap S_{\beta}$ is a half ball contained in $S_{\beta}$. It is easy to see that if $\beta>\pi$ (i.e., $S_{\beta}$ is not convex) then

$$
P_{S_{\beta}}(D)=n\left(\frac{C_{n}}{2}\right)^{1 / n}|D|^{(n-1) / n}<n \beta_{n}^{1 / n}|D|^{(n-1) / n},
$$

where

$$
\beta_{n}=\left|S_{\beta} \cap B_{1}\right| \text {. }
$$

In this example the solid angle of the cone $S_{\beta}$ is larger than $\omega_{n-1} / 2$. It is possible to construct nonconvex cones $\Sigma_{\alpha}$ for which the isoperimetric inequality (1.1) does not hold even if the solid angle $\alpha$ is very small. An example could be the following:

Let $A_{1}$ and $A_{2}$ be two disjoint subsets of $S^{n-1}$ with $H_{n-1}\left(A_{1}\right)=\alpha^{1}<$ $H_{n-1}\left(A_{2}\right)=\alpha^{2}$. Let us denote by $A_{3}$ a very "thin" subset of $S^{n-1}$ connecting $A_{1}$ and $A_{2}$ with $H_{n-1}\left(A_{3}\right)=\alpha^{3}>0, \alpha^{3}$ being obviously very small. We consider the nonconvex cone $\Sigma_{\alpha}=\bigcup_{i=1}^{3} \Sigma_{\alpha^{i}}, \alpha=\sum_{i=1}^{3} \alpha^{i}$ and the sector $D=$ $\Sigma\left(\alpha^{1}, R\right), R>0$, homothetic to $\Sigma_{\alpha^{1}}$. Since the cone $\Sigma_{\alpha^{3}}$ is very thin there exists $\varepsilon>0$, so small that

$$
P_{\Sigma_{\alpha}}(D)=\left(n\left(\alpha_{n}^{1}\right)^{1 / n}+\varepsilon\right)|D|^{(n-1) / n}<n \alpha_{n}^{1 / n}|D|^{(n-1) / n}
$$

which contradicts the isoperimetric inequality (1.1).

Obviously $\alpha^{1}$ and $\alpha^{2}$ may be chosen arbitrarily small (in particular we can have $\left.\alpha=\sum_{i=1}^{3} \alpha^{i}<\omega_{(n-1) / 2}\right)$. Therefore this second counterexample shows that the amplitude of the solid angle corresponding to $\Sigma_{\alpha}$ does not play a role in the isoperimetric inequality (1.1).

2. In this section we briefly indicate how Theorem 1.1 can be applied to extend the results of [15] and [17] to all convex sectors.

Let $\Omega$ be a bounded domain in $\mathbf{R}^{n}$ whose boundary is lipschitz continuous and is made of two manifolds $\Gamma_{0}$ and $\Gamma_{1}$. The "isoperimetric constant of $\Omega$ relative to $\Gamma_{1} ", Q\left(\Gamma_{1}, \Omega\right)$ is defined by [17]

$$
Q\left(\Gamma_{1}, \Omega\right)=\sup _{E} \frac{|E|^{1-1 / n}}{P_{\Omega}(E)},
$$

where the supremum is taken over all measurable subsets $E$ of $\Omega$ such that $\partial E \cap \Gamma_{0}$ does not contain any set of positive $(n-1)$-dimensional Hausdorff measure.

Several properties of $Q\left(\Gamma_{1}, \Omega\right)$ are listed in [15] and [17]. We just recall here that if both $\Gamma_{0}$ and $\Gamma_{1}$ have positive $(n-1)$-dimensional Hausdorff measure then $Q\left(\Gamma_{1}, \Omega\right) \in\left[\left(n\left(C_{n} / 2\right)^{1 / n}\right)^{-1},+\infty\right]$. So if $Q\left(\Gamma_{1}, \Omega\right)<+\infty$, there exists 
a number $\left.\left.\alpha_{n} \in\right] 0, C_{n} / 2\right]$ such that $Q\left(\Gamma_{1}, \Omega\right)=\left(n \alpha_{n}^{1 / n}\right)^{-1}$ and, obviously, $\alpha_{n}$ can be considered as the measure of a unitary sector $\Sigma(\alpha, 1)$.

Given a sector $\Sigma(\alpha, R)$, let us set $\widetilde{\Gamma}_{0}=\{x \in \partial \Sigma(\alpha, R),|x|=R\}$ and $\widetilde{\Gamma}_{1}=\partial \Sigma(\alpha, R) \backslash \widetilde{\Gamma}_{0}$.

The main consequence of Theorem 1.1 is the following:

Proposition 2.1. If $\Sigma(\alpha, R)$ is a convex cone then $Q\left(\widetilde{\Gamma}_{1}, \Sigma(\alpha, R)\right)$ is a maximum and

$$
Q\left(\widetilde{\Gamma}_{1}, \Sigma(\alpha, R)\right)=\left(n \alpha_{n}^{1 / n}\right)^{-1}=\frac{|\Sigma(\alpha, r)|^{(n-1) / n}}{H_{n-1}\{x \in \Sigma(\alpha, R):|x|=r\}}
$$

for any sector $\Sigma(\alpha, r) \subset \Sigma(\alpha, R), 0<r<R$.

Remark 2.1. If $\partial \Sigma_{\alpha} \backslash\{P\}$ is smooth, in view of the uniqueness statement of Theorem 1.1, we can also say that the sector $\Sigma(\alpha, r), r \in] 0, R[$, are the only sets which realize the maximum $Q\left(\widetilde{\Gamma}_{1}, \Sigma(\alpha, R)\right)$.

The definition of the isoperimetric constant $Q\left(\Gamma_{1}, \Omega\right)$ allows us to collect the domains $\Omega$ of the type considered above in classes $\mathscr{E}_{\alpha_{n}}$ defined as $\mathscr{E}_{\alpha_{n}}=\{\Omega$ such that $\left.Q\left(\Gamma_{1}, \Omega\right)=\left(n \alpha_{n}^{1 / n}\right)^{-1}\right\}$. Moreover Proposition 2.1 implies that any convex sector $\Sigma(\alpha, R)$ belongs to the class $\mathscr{E}_{\alpha_{n}}, \alpha_{n}=|\Sigma(\alpha, 1)|$.

The classes $\mathscr{E}_{\alpha_{n}}$ were introduced in [15] and [17] to study the symmetrization of functions $u \in V^{p}(\Omega), V^{p}(\Omega)=\left\{u \in H^{1, p}(\Omega), u=0\right.$ on $\left.\Gamma_{0}\right\}$. To be more precise if $\Omega \in \mathscr{E}_{\alpha_{n}}$ and $C_{\alpha}(\Omega)$ is a convex sector in $\mathscr{E}_{\alpha_{n}}$ with $\left|C_{\alpha}(\Omega)\right|=|\Omega|$, then the $\alpha$-symmetrization is defined as the transformation which associates to any $u \in V^{p}(\Omega)$ the unique radial decreasing function $C_{\alpha}(u) \in V^{p}\left(C_{\alpha}(\Omega)\right)$ having the same distribution function as $u[3,15,17]$.

If $\Omega \in \mathscr{E}_{\alpha_{n}}$ then the $\alpha$-symmetrization has the usual properties of the Schwarz symmetrization.

Let us remark explicitly that because of the isoperimetric property of the convex sectors (Theorem 1.1 and hence Proposition 2.1) any radial decreasing function $u \in V^{p}(\Sigma(\alpha, R)), \Sigma(\alpha, R)$ being a convex sector, stays fixed under the $\alpha$-symmetrization. Thus, in the $\alpha$-symmetrization the convex sectors play the same role as the ball in the Schwarz symmetrization.

Since the functions $u \in V^{p}(\Omega)$ are zero only on a part of $\partial \Omega$, namely $\Gamma_{0}$, the $\alpha$-symmetrization is suitable to study partial differential equations with mixed boundary conditions. A model problem of this type has been considered in [17, Theorem 2.1]. Actually, in view of Theorem 1.1 and Remark 2.1 we can state a slightly improved result.

Proposition 2.2. Let $\Sigma_{\alpha}$ be a convex cone and $\Omega$ a bounded domain in $\Sigma_{\alpha}$. Assume that $\partial \Sigma_{\alpha}$ and $\partial \Omega$ are both lipschitz continuous and $H_{n-1}\left(\partial \Sigma_{\alpha} \cap \partial \Omega\right)>0$. 
Let $\Gamma_{1}=\partial \Sigma_{\alpha} \cap \partial \Omega, \Gamma_{0}=\partial \Omega \backslash \Gamma_{1}$ and $u$ be a weak solution of the problem

$$
\begin{cases}-\sum_{i, j=1}^{n} D_{x_{i}}\left(a_{i j}(x) D_{x_{j}} u\right)=f & \text { in } \Omega, \\ u=0 & \text { on } \Gamma_{0}, \\ \frac{\partial u}{\partial \nu}=0 & \text { on } \Gamma_{1},\end{cases}
$$

where $\nu$ is the outer normal to $\Gamma_{1}, f \in L^{t}(\Omega)(t=2 n /(n+2)$ if $n>2, t>1$ if $n=2)$ and $a_{i j} \in L^{\infty}(\Omega)$ satisfy

$$
\sum_{i, j=1}^{n} a_{i j}(x) \xi_{i} \xi_{j} \geq|\xi|^{2} \quad \text { for every } \xi \in \mathbf{R}^{n} .
$$

Then, if $\Omega \in \mathscr{E}_{\alpha_{n}}$ we have

$$
C_{\alpha}(u) \leq v(x) \quad \text { pointwise in } C_{\alpha}(\Omega)=\left\{x \in \Sigma_{\alpha},|x|<R_{\Omega}\right\}
$$

where $v(x)$ is the weak solution of

$$
\begin{cases}-\Delta v=C_{\alpha}(f) & \text { in } C_{\alpha}(\Omega), \\ v=0 & \text { on } \widetilde{\Gamma}_{0}=\left\{x \in \Sigma_{\alpha},|x|=R_{\Omega}\right\}, \\ \frac{\partial v}{\partial \nu}=0 & \text { on } \widetilde{\Gamma}_{1}=\partial C_{\alpha}(\Omega) \backslash \widetilde{\Gamma}_{0} .\end{cases}
$$

Moreover if $\partial \Sigma_{\alpha} \backslash P$ is smooth and $C_{\alpha}(u)=v$ almost everywhere in $C_{\alpha}(\Omega)$, necessarily $\Omega=C_{\alpha}(\Omega), f=C_{\alpha}(f)$ and $u=v$.

The novelty of this result, compared to that of [17, Theorem 2.1] is that it holds for any convex cone and if $\partial \Sigma_{\alpha} \backslash P$ is smooth the inequality (2.5) is strict a.e. unless $\Omega$ is itself a convex sector homothetic to $\Sigma_{\alpha}$. The proof of this last statement is the same as that of [1, Theorem 1].

Another application of the $\alpha$-symmetrization has been given in [15] in estimating the best Sobolev constant $S^{p}(\Omega)$ for the imbedding $V^{p}(\Omega) \hookrightarrow L^{q}(\Omega)$, $q=n p /(n-p), n \geq 3$. Also in this case, the isoperimetric property of the convex sectors of Theorem 1.1 allows us to improve the results of [15], stated only for a certain type of convex sectors.

In particular, from [15, Theorem 2.1] and Proposition 2.1 here, we deduce the following:

Proposition 2.3. If $\Sigma(\alpha, R)$ is a convex sector then

$$
\begin{aligned}
S^{p}(\Sigma(\alpha, R)) & =\inf _{\substack{u \in V^{p}(\Sigma(\alpha, R)) \\
u \neq 0}} \frac{\|D u\|_{p}}{\|u\|_{q}}=\inf _{\substack{u \in \bar{V}^{p}(\Sigma(\alpha, R)) \\
u \neq 0}} \frac{\|D u\|_{p}}{\|u\|_{q}} \\
& =\bar{S}^{p}(\Sigma(\alpha, R)),
\end{aligned}
$$

where

$$
\bar{V}^{p}(\Sigma(\alpha, R))=\left\{u \in V^{p}(\Sigma(\alpha, R)) ; u \text { is radial }\right\}
$$


In other words Proposition 2.3 states that if $\Sigma(\alpha, R)$ is a convex sector the best Sobolev constant $S^{p}(\Sigma(\alpha, R))$ can be obtained by a minimizing sequence made of spherically symmetric functions. Once again we remark that this "symmetry property" of $S^{p}(\Sigma(\alpha, R))$ is a direct consequence of the isoperimetric property of the convex sectors.

Then it is natural to ask whether this symmetry property is preserved also for solutions of semilinear elliptic equations in convex sectors, as it happens in the case of the ball (see [9]). A positive answer to this problem is given in [4] where only a particular case of convex sectors is considered (namely the ones considered in [15]). The proof of this symmetry result given in [4] however does not use any isoperimetric inequality or the $\alpha$-symmetrization. Nevertheless, in view of Theorem 1.1 it is reasonable to conjecture that the symmetry result of [4] should extend to positive solutions of semilinear elliptic equations in any convex sector.

\section{ACKNOWLEDGMENTS}

We wish to thank P. Bérard, E. De Giorgi and S. Gallot for some useful discussions about the subject of this note and for having pointed out some useful references.

\section{REFERENCES}

1. A. Alvino, P. L. Lions, and G. Trombetti, A remark on comparison results via symmetrization, Proceed. Royal Soc. Edinburgh 102A (1986), 37-48.

2. G. Anzellotti, M. Giaquinta, U. Massari, G. Modica, and L. Pepe, Note sul problema di Plateau, Pisa, Editrice Tecnico Scientifica, 1974.

3. C. Bandle, Isoperimetric inequalities and applications, Pitman, London, 1980.

4. H. Berestycki and F. Pacella, Symmetry properties for positive solutions of elliptic equations with mixed boundary conditions, J. Funct. Anal. (1) 87 (1989), 177-211.

5. E. De Giorgi, Su una teoria generale della misura $(r-1)$-dimensional in uno spazio a $r$ dimensioni, Ann. Mat. Pura Appl. (4) 36 (1954), 191-213.

6. __ Sulla proprietà isoperimetrica della ipersfera nella classe degli insiemi aventi frontiera orientata di misura finita, Mem. Accad. Naz. Lincei (8) 5 (1958), 33-44.

7. H. Federer, Geometric measure theory, Springer-Verlag, 1969.

8. S. Gallot, Some links between isoperimetry, spectrum and topology, in preparation.

9. B. Gidas, W. N. Ni, and L. Nirenberg, Symmetry and related properties via the maximum principle, Comm. Math. Phys. 68 (1979), 209-243.

10. E. Giusti, The equilibrium configuration of liquid drops, J. Reine Angew. Math. 321 (1981), 53-63.

11. E. Gonzalez and G. Greco, Una nuova dimostrazione della proprietà isoperimetrica dell 'ipersfera nella classe degli insiemi aventi perimetro finito, Ann. Univ. Ferrara 23 (1977), 251-256.

12. E. Gonzalez, U. Massari, and I. Tamanini, On the regularity of boundaries of sets minimizing perimeter with a volume constraint, Indiana Univ. Math. J. 32 (1983), 25-37.

13. M. Gromov, Paul Levy's isoperimetric inequality, Prépublication I.H.E.S., 1980.

14. H. Hadwiger, Vorlesungen über inhalt, oberfläche und isoperimetric, Springer-Verlag, 1957.

15. P. L. Lions, F. Pacella, and M. Tricarico, Best constants in Sobolev inequalities for functions vanishing on some part of the boundary and realted questions, Indiana Univ. Math. J., 37 (1988), 301-324. 
16. V. G. Maz'ja, Sobolev spaces, Springer-Verlag, 1985.

17. F. Pacella and M. Tricarico, Symmetrization for a class of elliptic equations with mixed boundary conditions, Atti Sem. Mat. Fis. Univ. Modena XXXIV (1985-1986), 75-94.

18. G. Polya and G. Szegö, Isoperimetric inequalities in mathematical physics, Ann. of Math. Studies, Princeton, 1951.

Ceremade, Université Paris-Dauphine, Place de Lattre de Tassigny, 75775 Paris Cedex 16, FRANCE

Dipartimento di Matematica, Universitá di Rome "La Sapienza", Piazzale A. Moro 2, 00185 Roma, Italia 